\title{
The Pierre Auger Observatory status and latest results
}

\author{
Corinne Berat ${ }^{1, *}$ for the Pierre Auger Collaboration ${ }^{2, * *}$ \\ ${ }^{1}$ LPSC, Université Grenoble-Alpes, CNRS/IN2P3, Grenoble, France \\ ${ }^{2}$ Observatorio Pierre Auger, Av. San Martìn Norte 304, 5613 Malargüe, Argentina
}

\begin{abstract}
Ultra-high energy cosmic rays represent the most energetic particles available to scientists. These particles have macroscopic energies, exceeding $1 \mathrm{EeV}$, but their detection is a challenge, their flux being very weak, around one particle per square kilometer per century for the highest energies. The Pierre Auger Observatory, in Argentina, is the present flagship experiment studying ultra-high energy cosmic rays. The combination of a large array of surface detectors covering $3000 \mathrm{~km}^{2}$ and fluorescence telescopes enhances the performances of the extensive air shower detection and measurements, resulting in both high statistics and unprecedented data quality. Moreover, the operation of a denser sub-array has extended the sensitivity to lower energies. Altogether, these well performing detectors contribute to providing important information on key questions on cosmic rays in the energy range from $0.1 \mathrm{EeV}$ up to $100 \mathrm{EeV}$, as highlighted in this presentation of the latest results obtained by the Pierre Auger Collaboration. Despite a large number of valuable results, the understanding of the nature and the origin of the highest energy cosmic rays remains an open science case that the Auger Collaboration is willing to address with the AugerPrime project.
\end{abstract}

\section{Introduction}

In the extreme part of the charged cosmic-ray spectrum, the Ultra-High Energy Cosmic Rays (UHECR) have macroscopic energies and represent the most energetic source of elementary particles available to scientists. Some of these cosmic particles are a million times more energetic than those produced at the CERN's Large Hadron Collider, the most powerful particle accelerator on Earth. Unfortunately, their flux is as low as one particle per square kilometer per century. Above $10^{14} \mathrm{eV}$, high-energy cosmic rays entering the Earth's atmosphere initiate cascades of secondary particles and produce extensive air showers (EAS) that extend sufficiently to reach the ground. At $10 \mathrm{EeV}$, an EAS contains $\sim 10^{10}$ particles co-moving within a thin disc at velocities close to the speed of light spreading over an area of $\sim 20 \mathrm{~km}^{2}$. An EAS contains an electromagnetic component (electrons, positrons, and photons) and a muonic component that can be sampled using ground arrays of particle detectors. Charged particles in the shower also excite nitrogen molecules in the air, producing fluorescence light that can be observed with telescopes during clear nights. The Pierre Auger Observatory, described in section 2, is dedicated to studying UHECR based on measurements of air-shower observables. The main and latest results obtained from the collected data are presented in

\footnotetext{
*e-mail: berat@lpsc.in2p3.fr

**e-mail: auger_spokespersons@fnal.gov _ Full author list: http://www.auger.org/archive/authors_2018_09.html
} 
section 3. The AugerPrime project and the promising future of the Pierre Auger Observatory are described in the last section.

\section{Pierre Auger Observatory}

The Pierre Auger Observatory [1] is located in the province of Mendoza (Argentina) and covers 3000 square kilometers (Fig. 1-left). It uses two proven techniques to measure the properties of extensive air showers by observing their lateral spread at ground level with a Surface Detector (SD), as well as their longitudinal development in the atmosphere with a Fluorescence Detector (FD).

To detect charged particles and photons that reach the ground, the SD consists in 1660 autonomous water-Cherenkov detectors (WCD), filled with 12 tonnes of pure water and equipped with three photomultipliers each. The WCD are distributed on a triangular grid with a spacing of $1500 \mathrm{~m}$, except for $61 \mathrm{WCD}$ forming a denser array of $23.5 \mathrm{~km}^{2}$ on a $750 \mathrm{~m}$ grid. The surface detector operates 24 hours per day, and provides a huge collecting area. The fluorescence light produced along the path of the showers above the surface detector is observed by the FD, consisting of 27 fluorescence telescopes. Twenty four telescopes have a field of view of $30^{\circ} \times 30^{\circ}$ in azimuth and elevation, with a minimum elevation of $1.51^{\circ}$ above the horizon. Three additional telescopes, the High Elevation Auger Telescopes, can cover an elevation up to $60^{\circ}$ to detect the low-energy showers in coincidence with the dense array. Light is focused with a spherical mirror of $11 \mathrm{~m}^{2}$ effective area on a camera of 440 hexagonal PMTs. The FD can only operate in dark, moonless nights with a field of view free of clouds. This limits its duty cycle to $\sim 15 \%$. The atmosphere acting as a calorimeter for the FD measurements, has to be surveyed accurately. Hence, the Observatory includes a set of high-quality devices for monitoring clouds and aerosols during operation, such as a central laser facility (CLF), an extreme laser facility (XLF), a robotic photometric telescope FRAM, lidars, and IR cloud cameras [3].

The longitudinal profile reconstructed by the FD is providing a nearly calorimetric measurement of the primary energy, with total systematic uncertainty of 14\% [4]. It also allows determining the depth of maximum of the shower development $X_{\max }$ with a resolution of $20 \mathrm{~g} \mathrm{~cm}^{-2}$. From the shower lateral distribution reconstructed using the WCD signals, an SD energy estimator is inferred. The hybrid detection system allows calibrating the SD energy estimator by exploiting a high-quality subset of events recorded simultaneously by the FD and at least one WCD of the SD using the FD energy measurement, hence providing an almost model-independent energy calibration. The particle arrival time is used to infer the arrival direction of the showers. The angular resolution achieved for events reconstructed with more than three WCD is better than $1.6^{\circ}$, and better than $0.9^{\circ}$ for events with more than six WCD.

Two more detector types are employed within the Pierre Auger Observatory site. A $17 \mathrm{~km}^{2}$ sub-array of 153 radio sensors (Auger Engineering Radio Array) [5] is exploiting the coherent radiation in EAS $(30-80 \mathrm{MHz})$, which is sensitive to the longitudinal development of the shower. The Auger Muon and Infill for the Ground Array (AMIGA) [6] consists of scintillator panels of $10 \mathrm{~m}^{2}$ area buried besides 61 WCDs of the denser array to detect the muon content of EAS.

\section{Latest results}

High-quality data have been collected continuously for more than ten years, with an SD annual exposure of $\sim 5500 \mathrm{~km}^{2} \mathrm{sr}$ yr, leading to a wealth of important and valuable results; a selection among them are presented below. 


\subsection{Energy spectrum}
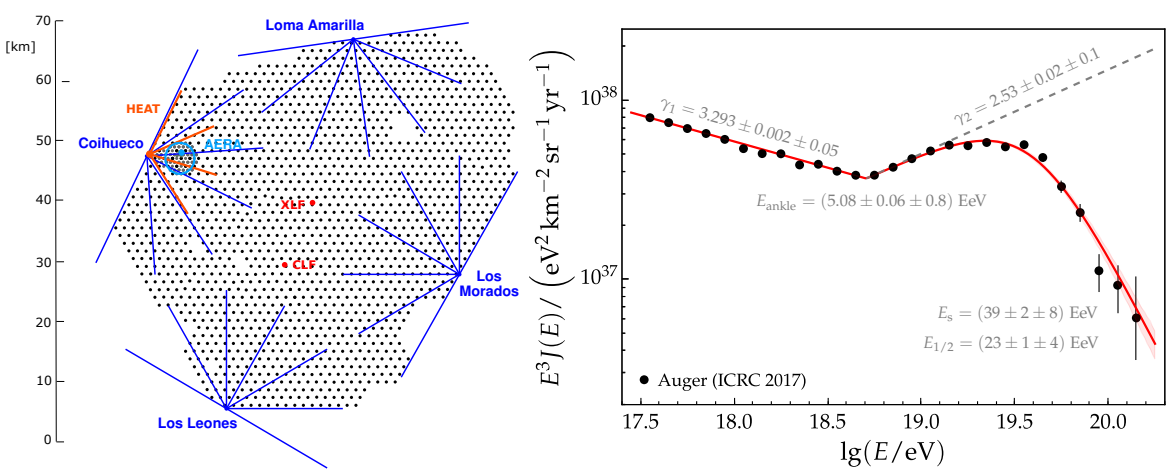

Figure 1. Left: layout of the Pierre Auger Observatory: black dots indicate WCD positions, blue and orange lines show the azimuthal field of view of the fluorescence telescopes [2]. Right: the combined energy spectrum and the fitting function with the fitting parameters [7].

The accurate measurement of the energy spectrum is required for astrophysical modeling of the origin, the nature and the propagation of UHECRs. The latest measurements [7] benefit from an improved reconstruction of the FD and SD events, and four different spectra are reconstructed, each of them based on a specific dataset. All the spectra agree within the systematic uncertainties, which are dominated by the one in the energy scale (14\%). The systematic uncertainties on the flux are between 5 and $10 \%$ and are responsible for the difference in normalization between the spectra. The four independent measurements are combined to get the UHECR spectrum using a method that takes into account the systematic uncertainties of the individual measurements. The result presented on Fig. 1-right is based on an exposure larger than $67000 \mathrm{~km}^{2} \mathrm{sr} \mathrm{yr}$, allowing for a determination of the spectrum features with an unprecedented precision and to probe the spectrum up to $10^{20.15} \mathrm{eV}$.

The combined spectrum is fitted with a power law allowing for a break in the spectral index at $E_{\text {ankle }}$ and a smooth exponential suppression to describe the behavior at the highest energies. At $E_{\text {ankle }}=(5.08 \pm 0.06$ (stat) \pm 0.8 (syst) $) \mathrm{EeV}$, the spectral index hardens from $\gamma=$ 3.29 to $\gamma=2.53$. The measured spectrum excludes a power-law extension of the flux above the ankle towards ultra-high energies and the suppression energy is found at $E_{\mathrm{s}}=39 \pm 2$ (stat) \pm 8 (syst) EeV. The origin of the suppression could be due to propagation effects (Greisen, Zatsepin and Kuz'min (GZK) cutoff [8]) or to the reach of the maximum accelerating energy attainable at the source (or to a combination of both effects). Information on the primary nature is necessary to interpret the end of the CR spectrum. The energy value at which the integral flux drops by a factor of two below what would be expected without suppression is found to be $E_{1 / 2}=(23 \pm 1$ (stat) \pm 4 (syst) $\mathrm{EeV}$ disfavoring the scenario of a flux of protons produced uniformly in extragalactic space suffering energy losses due to the GZK effect. Indeed, in this type of scenario, the expected value for $E_{1 / 2}$ is $53 \mathrm{EeV}$.

\subsection{Anisotropy searches}

The distribution of UHECR arrival directions are scrutinized to get information on their origin. Cosmic rays being charged particles, they interact with both the magnetic fields in our Galaxy and the intergalactic medium that lies between the sources and Earth, and can be 
Table 1. Data from the Rayleigh analysis of the first harmonic in right ascension for the two energy bins [9].

\begin{tabular}{|c|c|c|c|c|}
\hline $\mathrm{E}[\mathrm{EeV}]$ & events & amplitude $r$ & phase [deg.] & $\mathrm{P}(\leq r)$ \\
\hline $4-8$ & 81701 & $0.005_{-0.002}^{+0.006}$ & $80 \pm 60$ & 0.60 \\
$>8$ & 32187 & $0.047_{-0.007}^{+0.008}$ & $100 \pm 10$ & $2.610^{-8}$ \\
\hline
\end{tabular}
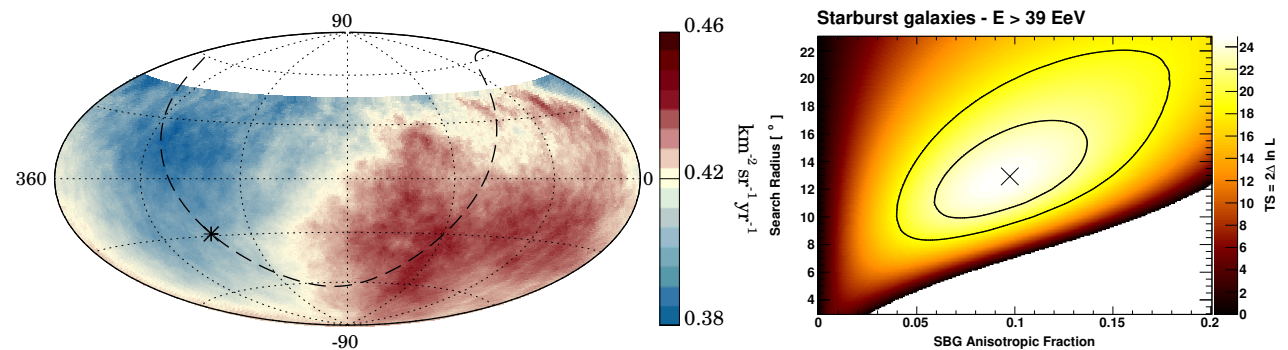

Figure 2. Left: Map showing the cosmic-ray flux above $8 \mathrm{EeV}$ in equatorial coordinates. The galactic plane (galactic center) is shown by a dashed line (asterisk) [9]. Right: TS profile above $39 \mathrm{EeV}$ over the fit parameters for SBG model; the lines indicate the $1 \sigma$ and $2 \sigma$ regions [10] .

strongly deflected. Therefore, it is difficult to locate their sources. However, anisotropies in the distribution of their arrival directions may be detectable on large angular scales. The observation of a large-scale anisotropy in the arrival directions of UHECRs above $8 \mathrm{EeV}$ was reported by the Auger Collaboration in Science [9] in 2017. The dataset corresponds to more than ten years of data, and a total exposure of $76800 \mathrm{~km}^{2} \mathrm{sr} \mathrm{yr}$, in a declination range of $-90^{\circ}<\delta<+45^{\circ}$ (85\% sky coverage). Showers with a zenith angle below $80^{\circ}$ and an energy above $4 \mathrm{EeV}$ (full trigger efficiency threshold, angular resolution better than $1^{\circ}$ ) are selected, and corrections are applied to eliminate any spurious systematics effects induced by the atmospheric variations and the geomagnetic field.

Analyses rely on a weighted harmonic expansion of the cosmic-ray flux distribution, in which the weights take into account the directional exposure and the slight tilt of the SD array. Events were divided into two energy bins $4 \mathrm{EeV}<E<8 \mathrm{EeV}$ and $E>8 \mathrm{EeV}$. The results of the harmonic analysis in right ascension are presented in Table 1 for each energy bin. With the amplitude and the phase derived from the analysis are given the probabilities that amplitudes larger than those observed could arise by chance from fluctuations in an isotropic distribution. In the first energy bin, the results are consistent with an isotropic distribution, but for $E>8 \mathrm{EeV}$, a significant modulation is observed $(5.2 \sigma)$. The results of the Rayleigh analysis in right ascension are compatible with a dipolar modulation, but this analysis is only sensitive to the dipole component in the equatorial plane. The distribution of the azimuth angles being sensitive to the component parallel to the Earth's rotation axis, an harmonic analysis in azimuth angle is performed, allowing the $3 \mathrm{D}$ dipole reconstruction. The reconstructed dipole has an amplitude of $0.065_{-0.009}^{+0.013}$ and is pointing to $(\alpha, \delta)=(100,-24)$ (Fig. 2-left). That direction lies $\simeq 125^{\circ}$ from the Galactic Center, suggesting an extragalactic origin of these UHECR above $8 \mathrm{EeV}$.

Only a few source classes could be capable of accelerating charged nuclei up to ultra-high energy, and inhomogeneities in their spatial distribution may imprint anisotropy on a smaller angular scale, which can be revealed by comparing measured UHECR arrival directions with position of astrophysical objects. Given a model of candidate sources, a probability map of 

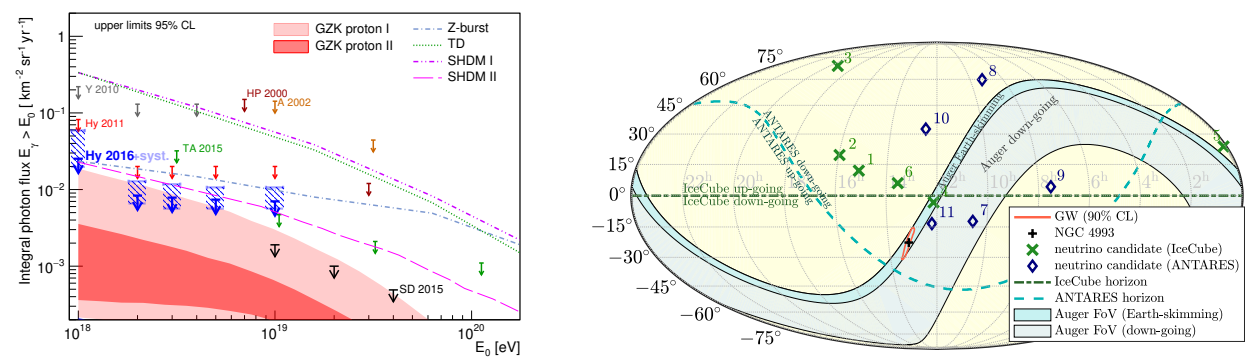

Figure 3. Left : Upper limits on the diffuse photon compared to limits from other experiments and model predictions; see [11] for references. Right: Localizations and sensitive sky areas at the time of the GW event in equatorial coordinates: GW 90\% credible-level localization (red contour); see [13] for details and references. Auger's fields of view for Earth-skimming and down-going neutrinos are indicated in dark and lighter blue respectively.

the arrival distribution of cosmic rays can be obtained in the form of a smoothed density map and compared to the observed distribution of events using an appropriate test statistic (TS).

In a recently published study [10] such a comparison was done, with a focus on $\gamma$-ray sources from Fermi-LAT catalogs. One type of sources are the radio loud AGN from the 2FHL catalog (Fermi-LAT, 360 sources), from which 17 within $250 \mathrm{Mpc}$ were extracted, selected according to their $\gamma$-ray flux above $50 \mathrm{GeV}$. A second class is composed of the starburst galaxies (SBGs), 63 objects within the same distance mentioned before, among which 23 with a flux larger than 0.3 Jy were selected. For each case, a sky model is built using an isotropic component plus an anisotropic contribution from $\gamma$-ray sources. The smoothed maps are constructed by weighting the objects by their relative flux, the $\gamma$-ray and the radio flux for the AGNs and the starburst galaxies respectively. The free parameters of the model are the fraction of anisotropy and the search radius. Events above $20 \mathrm{EeV}$ and with a zenith angle up to $80^{\circ}$ are used in this analysis, corresponding to a total exposure of $\sim 90000 \mathrm{~km}^{2}$ sr $\mathrm{yr}$ and a declination between $-90^{\circ}$ and $+45^{\circ}$. The TS is a likelihood ratio test between two hypotheses: the considered sky model and an isotropic model (null hypothesis). The TS is maximized as a function of the two model parameters. A scan in energy was done in order to determine the threshold energy for maximum TS. The best significance $(4.0 \sigma)$ is reached when the test hypothesis of SBGs plus isotropic distribution is tested against the null hypothesis (isotropy) for a threshold energy of $39 \mathrm{EeV}$. The optimal fit parameters are $10 \%$ of SBG correlated cosmic rays and $13^{\circ}$ search radius (Fig. 2-right).

\subsection{Neutral cosmic messengers}

Ultra-high energy neutrinos and photons are expected to be produced by UHECR interactions at the production site and during their propagation via the GZK process, i.e. the interactions of UHE protons with the CMB photons $\mathrm{p}+\gamma_{\mathrm{CMB}} \rightarrow \mathrm{p}+\pi^{0}, \pi^{0} \rightarrow \gamma \gamma, \mathrm{p}+\gamma_{\mathrm{CMB}} \rightarrow \mathrm{n}+\pi^{+}$, $\pi^{+} \rightarrow \mu^{+}+v+\ldots$. The discovery of these UHE particles could help to answer fundamental questions about UHE cosmic rays.

The Pierre Auger Observatory is a key detector in the field of multi-messenger astrophysics, being capable to discriminate photons $(E>1 \mathrm{EeV})$ and neutrinos $(E>0.1 \mathrm{EeV})$ in the background of charged cosmic particles at EeV energies. On average, air showers initiated by UHE photons develop deeper in the atmosphere than showers of the same primary 
energy induced by hadrons, due to the typically smaller multiplicity of electromagnetic interactions. Another key difference is the lower average number of muons in photon-induced air showers compared to air showers initiated by hadrons. The latest results have been obtained with a multivariate analysis combining observables from both the SD and the FD [11]. Three candidates compatible with background expectations are found. Assuming a differential flux $d N(E)=k E^{2}$ allows deriving limits on the UHE photon fraction, lowered by up to a factor 4 compared to previously published limits, due to the larger dataset used in this analysis and the improvements in the background rejection. The top-down scenarios proposed to explain the origin of UHE cosmic-ray production are severely constrained, and the achieved sensitivity allows testing photon fractions of about $0.1 \%$ and exploring the region of photon fluxes predicted in some astrophysical scenarios (indicated by the shaded areas in Fig. 3-left).

The neutrino search is performed by studying very inclined and earth-skimming showers [12]. Dedicated and efficient selection criteria are based on the characteristics expected for "young" showers initiated by neutrinos, developing deep in the atmosphere, compared to "old" ones from inclined hadronic showers, having their electromagnetic component fully absorbed before reaching the detectors. The corresponding exposure is determined by applying the same criteria to simulated neutrino-induced showers. Assuming a differential flux $d N(E)=k E^{2}$, upper limits are derived. The sensitivity to UHE neutrinos is maximum around $\mathrm{EeV}$ energies and allows to constrain models of cosmogenic neutrino production assuming that protons dominate at the sources.

The Observatory has a sensitivity to UHE neutrinos in a large fraction of sky from $-80^{\circ}$ to $60^{\circ}$ in equatorial declination, but is limited to large zenith angles, so that at each instant neutrinos can be efficiently detected only from a specific fraction of the sky. The neutronstar merger detected by LIGO-Virgo (GW170817) was fortunately in an optimal position for the detection of UHE tau neutrinos by Auger, since it was located in the field of view of the Earth-skimming channel at the instant of emission. Fig. 3-right shows the sensitive regions and summarizes the results of the search for high-energy neutrinos correlated with this event performed by the Pierre Auger Observatory together with the dedicated neutrino experiments IceCube and Antares [13]. No neutrino candidates directionally coincident with the merger were found within $\pm 500 \mathrm{~s}$ or within the 14-day period following the merger. This non-detection is consistent with model predictions of a short GRB observed off-axis. Nevertheless, the main message is that the Pierre Auger Observatory joined the common effort of numerous instruments and plays an active role in the new multi-messenger era in astronomy and astrophysics.

\subsection{Mass composition and hadronic interactions}

Knowledge on the composition of cosmic rays in the energy range of 0.1 to $1 \mathrm{EeV}$ is mandatory for a better understanding of the nature of the energy-spectrum features, and for identifying a possible transition from galactic to extra-galactic sources. The main observable sensitive to the mass of primary particle, $X_{\max }$, is directly obtained from the longitudinal profile measured by the FD. The $X_{\max }$ determination is based on data from the standard fluorescence detectors $\left(E>10^{17.8} \mathrm{eV}\right)$ complemented by the High Elevation Auger Telescopes ones, providing extension to low energies $(E>0.63 \mathrm{EeV})$. The measured evolution of $\left\langle X_{\max }\right\rangle\left(\sigma\left(X_{\max }\right)\right)$ with the primary energy is shown on Fig. 4-left (-right), as well as the evolutions predicted by the shower simulations using three different hadronic interaction models ${ }^{1}$ [15] [16] [17]. The measured $\left\langle X_{\max }\right\rangle$ increases by $79 \mathrm{~g} \mathrm{~cm}^{-2}$ per decade up to $2 \mathrm{EeV}$, where the rate of change becomes significantly smaller $\left(\sim 26 \mathrm{~g} \mathrm{~cm}^{-2}\right.$ per decade $)$. Air-shower

\footnotetext{
${ }^{1}$ the three hadronic interaction models used for the simulations have all been tuned to match data from the LHC.
} 

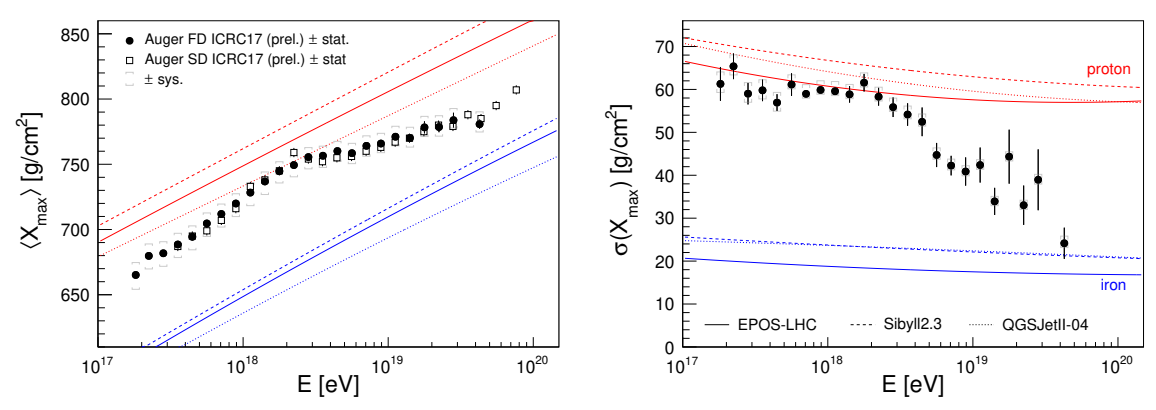

Figure 4. Measurements of $\left\langle X_{\max }\right\rangle$ (left) and $\sigma\left(X_{\max }\right)$ (right) as a function of the energy and predictions from simulations of proton- and iron-induced air showers [14].

simulations predict an increase of $\left\langle X_{\max }\right\rangle$ of $\sim 60 \mathrm{~g} \mathrm{~cm}^{-2}$ per decade, for any primary mass. The comparison indicates that the average mass of cosmic particles is getting lighter between 0.6 and $2 \mathrm{EeV}$, and then is becoming heavier. According to the values of $\sigma\left(X_{\max }\right)$ below $2 \mathrm{EeV}$, the data are compatible with a light composition or a mixed composition. At higher energy, the decrease of $\sigma\left(X_{\max }\right)$ indicates a trend to a heavier composition.

From $\left\langle X_{\max }\right\rangle$ and $\sigma\left(X_{\max }\right)$ it is possible to derive $\langle\ln A\rangle$ and $\sigma^{2}(\ln A)$, using the approach described in [18], the conversion being based on a parametrisation depending on hadronic interaction. For the three models, similar trends with energy are observed: $\langle\ln A\rangle$ is decreasing with energy and then around $2 \mathrm{EeV}$, the trend is reversed; $\sigma^{2}(\ln A)$ is almost constant until $2 \mathrm{EeV}$, then it starts to decrease. These results are an indication that the relative proton fraction becomes smaller for energies above $2 \mathrm{EeV}$. It has to be mentioned that the results obtained with QGSJETII-04 are at odds with the data, leading to unphysical values for $\sigma^{2}(\ln A)$.

Additional information on the composition can be obtained from the comparison of measured $X_{\max }$ distributions in bins of energy, with simulated ones. Different simulated samples are tested, varying the number of species and the mixing fractions, and using the three interaction models mentioned above. The collaboration has updated the study, and the results presented in [19], thanks to the extended energy range and the larger statistics (Fig. 5). A single elemental group is dominating the composition for each energy bin, starting from protons below the ankle and going through helium to nitrogen as the energy increases. Depending on the hadronic interaction model, a small proton fraction may persist up to ultra-high energies, and there might be an iron contribution emerging above $25 \mathrm{EeV}$. According to the fit performances, the mass fractions obtained with QGSJETII-04 should be interpreted with care.

An analysis in which the mass composition observable are derived from the SD data has been developped. The method relies on the study of the signal risetime in the WCD, leading to an observable correlated to $X_{\max }$, and the inferred results on mass composition published at the end of 2017 [20] confirmed those mentioned above.

These results on mass composition show that such studies could be used for testing the hadronic interaction models. It is also the case for the analyses of the muon component of the showers. The majority of muons in air showers are created in decays of charged pions. At ultra-high energies, it takes several generations of interactions until the average pion energy is sufficiently small for pion decay to dominate. As a result, the total number of muons in an air shower depends on details of hadronic interactions along a chain of interactions. Thus the muon content is an observable very sensitive to hadronic interaction models. Inclined events, 


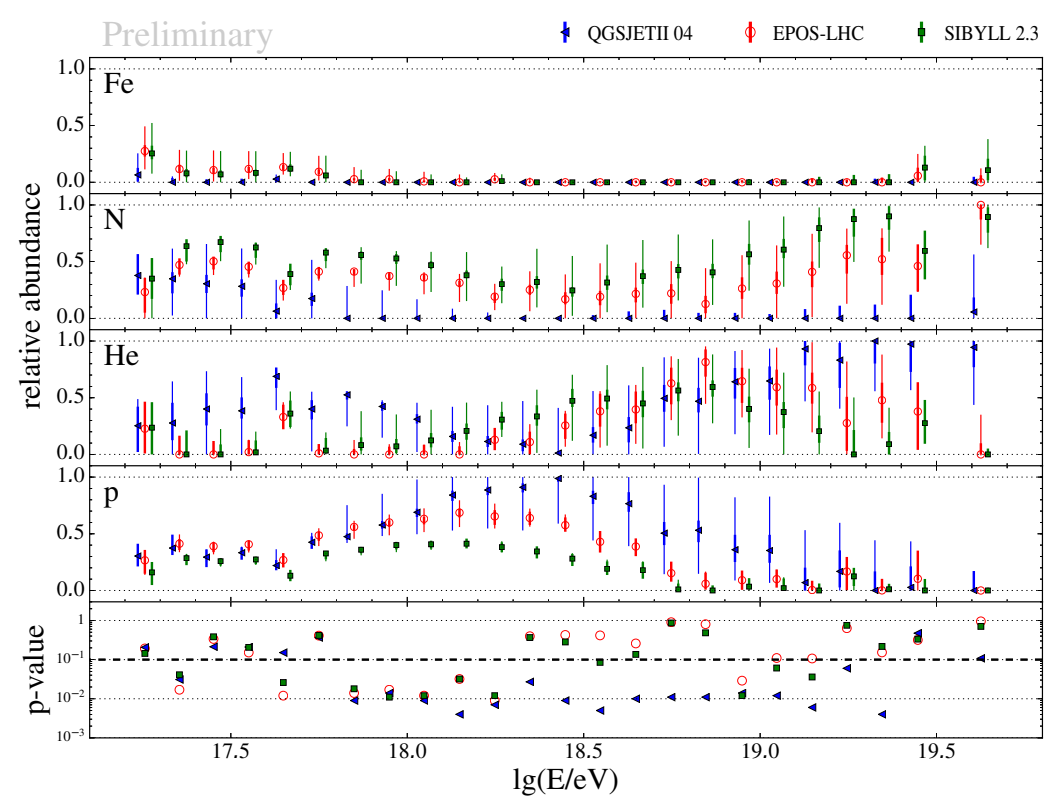

Figure 5. Results from a fit of the $X_{\max }$ distributions with a superposition of p-, He-, N- and Fe-induced air showers [19]. The upper four panels show the best-fit mass fractions and the goodness of fit is displayed in the lowest panel. Thick error bars denote the statistical uncertainties, thin error bars the systematic ones.

for which the electromagnetic component is largely absorbed before reaching the ground, have been used to measure the muon component [21], as well as hybrid events, for which the ground signal has been compared to the longitudinal profile [22]. All the performed analyses underline a muon deficit in simulations which could be more than $30 \%$ at $10^{19} \mathrm{eV}$ depending on the model.

\section{Outlook: AugerPrime}

The Pierre Auger Observatory has been successfully taking data since almost 15 years. Both the vast collection area and the hybrid detection strategy led to a rich harvest of valuable results, greatly improving our knowledge of the ultra-energetic cosmic rays, but leading to a complex and unexpected picture of UHECR, challenging the original expectation of few sources and light primaries. The existence of a flux suppression above $40 \mathrm{EeV}$ is incontestable, being due to propagation effect or to the reach of maximum energy at the sources. Evidence of anisotropies in the UHECR arrival directions emerge from several studies. At large scale, a dipole is observed, its direction indicating an extragalactic origin: it is a step forward in the understanding of the transitions between the galactic and extragalactic components. At intermediate scale, anisotropy is observed in association with starburst galaxies. With no UHE photon or neutrino found in data, limits on their fluxes are derived, clearly disfavoring "top-down" scenarios and leading to an astrophysical origin of UHECR, but no clear clue on any existing UHECR source has been revealed so far. The present photon limits also disfavor pure proton composition models. The image of a light and mixed composition around the ankle and a heavier composition at the highest energies is strengthened by different composition studies. Unfortunately, the $X_{\max }$ measurements above $40 \mathrm{EeV}$ with the 

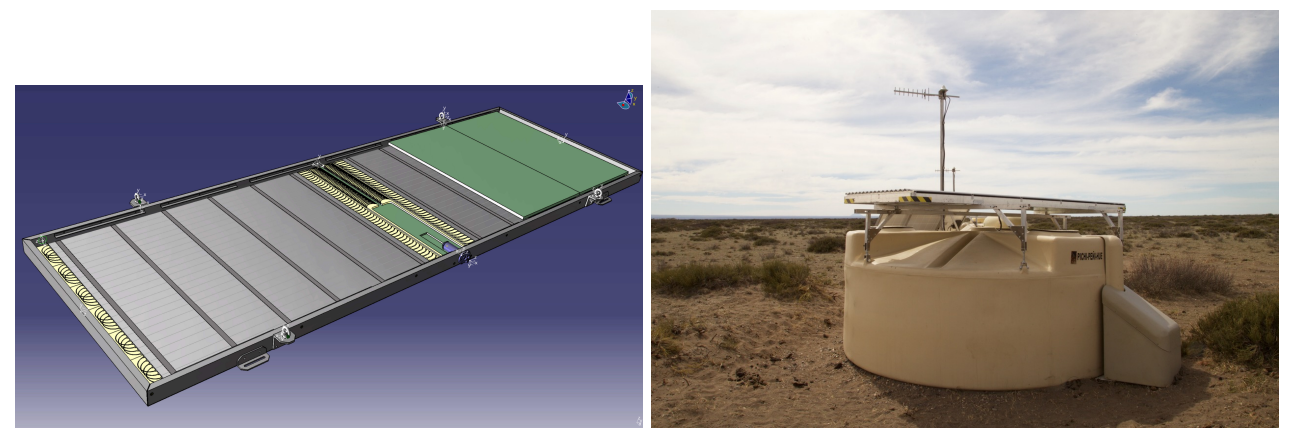

Figure 6. Left: the layout of the Surface Scintillator Detector; Right: one station of the AugerPrime Engineering Array [23].

FD are limited by its low duty cycle. But it is worthwhile to mention that mass composition studies using the SD data benefit of the $\sim 100 \%$ SD duty cycle, hence they allow extending the derived $X_{\max }$ measurements up to $10^{20} \mathrm{eV}$. The detailed interpretation of the data is currently limited by uncertainties in the hadronic interaction models, for which serious hints of deficiencies have been observed.

The astrophysical scenario resulting from Auger measurements is very complex and cannot at present be understood in terms of a unique interpretation for the sources, propagation and nature of the UHECRs. Information on the mass composition is crucial to go further in the interpretation of the observations, in particular at the highest energies. Moreover, discriminating between components would allow selecting light primaries pointing back to astrophysical sources, and would provide the unique opportunity to understand air-shower physics and hadronic multiparticle production (at center of mass energies well beyond the reach of LHC).

These questions are going to be addressed by the AugerPrime project [23], the Observatory upgrade program. It aims at improving the knowledge on mass composition, by discriminating electromagnetic and muonic components from SD-based observables, with a duty cycle of $\sim 100 \%$. The main component of the upgrade consists in equipping each WCD with a $3.8 \mathrm{~m}^{2}$ (1 cm thick) scintillating counter [24] installed on top of it (Fig. 6). The different sensitivity of the two detectors to the electromagnetic and muonic shower components of the shower will be used to disentangle between them. A new and improved electronics readout is developed [25], and the dynamic range of the SD detectors is extended by adding a smaller PMT [26] in each WCD to collect the Cherenkov light. Buried muon detectors in a sub-array [27] will allow validating the method of muonic and electromagnetic component estimation. In fall 2016, 12 scintillator counters were deployed in an Engineering Array [28], allowing the design to be finalized. The large-scale production of the scintillator started, and the deployment of new detectors is planed to start from fall 2018 for two years. The Observatory will then take data at least until 2025 in its upgraded configuration, aiming to answer the still open questions about UHECR nature and origin.

\section{References}

[1] The Pierre Auger Collaboration, Nucl. Instrum. Meth. A 798 (2015) 172 .

[2] M. Unger for the Pierre Auger Collaboration, Proc. of 35th ICRC, PoS (ICRC 2017) 1102. 
[3] The Pierre Auger Collaboration, J. Instrum. 7 (2012) P09001.

[4] V. Verzi for the Pierre Auger Collaboration, Proc. of 33rd ICRC, arXiv:1307.5059 (2013)

[5] The Pierre Auger Collaboration, Phys. Rev. Lett. 116, (2016) 241101.

[6] The Pierre Auger Collaboration, J. Instrum. 11 (2016), P02012.

[7] F. Fenu for the Pierre Auger Collaboration, Proc. of 35th ICRC, PoS (ICRC 2017) 486.

[8] K. Greisen, Phys. Rev. Lett. 16 (1966) 748.

G. T. Zatsepin, V. A. Kuz'min, J. Exp. Theor. Phys+ 4 (1966) 78.

[9] The Pierre Auger Collaboration, Science 357 (2017) 1266-1270.

[10] The Pierre Auger Collaboration, Astrophysical Journal Letters, 853:L29 (2018).

[11] The Pierre Auger Collab., JCAP 04 (2017) 009.

[12] E. Zas for the Pierre Auger Collaboration, Proc. of 35th ICRC, PoS (ICRC 2017) 972.

[13] ANTARES Collaboration, IceCube Collaboration, The Pierre Auger Collaboration, and LIGO Scientific Collaboration and Virgo Collaboration, Astrophysical Journal Letters, 850:L35 (2017).

[14] J. Bellido for the Pierre Auger Collaboration, Proc. of 35th ICRC, PoS (ICRC 2017) 506.

[15] S. Ostapchenko, Phys. Rev. D 83 (2011) 014018.

[16] T. Pierog et al. Phys. Rev. C 92 (2015) 034906.

[17] F. Riehn et al. Proc. of 34th ICRC, PoS (ICRC 2015) 558.

[18] The Pierre Auger Collaboration, JCAP 02 (2013) 026

[19] The Pierre Auger Collaboration, Phys. Rev. D 90, (2014) 122006.

[20] The Pierre Auger Collaboration, Phys. Rev. D 96, (2017) 122003.

[21] The Pierre Auger Collaboration, Phys. Rev. D 91, (2015) 032003.

[22] The Pierre Auger Collaboration, Phys. Rev. Lett. 117, (2016) 192001

[23] The Pierre Auger Collaboration,[arXiv:1604.03637].

D. Martello for the Pierre Auger Collaboration, Proc. of 35th ICRC, PoS (ICRC2017) 383.

[24] R. Smida for the Pierre Auger Collaboration, Proc. of 35th ICRC, PoS (ICRC2017) 390.

[25] T. Suomijarvi for the Pierre Auger Collaboration, Proc. of 35th ICRC, PoS (ICRC2017) 450.

[26] A. Castellina for the Pierre Auger Collaboration, Proc. of 35th ICRC, PoS (ICRC2017) 397.

[27] M.J. Figueira for the Pierre Auger Collaboration, Proc. of 35th ICRC, PoS (ICRC2017) 396.

[28] Z. Zong for the Pierre Auger Collaboration, Proc. of 35th ICRC, PoS (ICRC2017) 449. 